\title{
Casimir-Induced Instabilities at Metallic Surfaces and Interfaces
}

\author{
Kun Ding $\odot,{ }^{1,2}$ Daigo Oue, ${ }^{1}$ C. T. Chan, ${ }^{2, *}$ and J. B. Pendry ${ }^{1, \dagger}$ \\ ${ }^{1}$ The Blackett Laboratory, Department of Physics, Imperial College London, London SW7 2AZ, United Kingdom \\ ${ }^{2}$ Department of Physics, Hong Kong University of Science and Technology and Center for Metamaterials Research, \\ Clear Water Bay, Kowloon, Hong Kong, China
}

(Received 24 January 2020; accepted 8 January 2021; published 29 January 2021)

\begin{abstract}
Surface distortion splits surface plasmons asymmetrically in energy with a net lowering of zero-point energy. We contrast this with the symmetrical distortion of electronic energy levels. We use conformal mapping to demonstrate this splitting and find that surface corrugation always leads to a decrease in the zero-point energy of a metallic surface, but the decrease is not strong enough to drive a surface reconstruction on its own. A second metallic surface in proximity to the first gives a more significant lowering of energy, sufficient to drive the instability of a mercury thin film. This mechanism provides a fundamental length scale limit to planar nanostructures.
\end{abstract}

DOI: 10.1103/PhysRevLett.126.046802

Introduction.-Casimir forces [1,2] arise from zero-point quantum fluctuations of the electromagnetic field and play a central role in biology, adhesion, friction, wetting, and a host of other phenomena. Here we are concerned with the impact of Casimir forces on the stability of surfaces in the presence of distortion. Surface plasmons will be our focus. Let us contrast Casimir bonding with electronic bond formation. When two hydrogen atoms interact, their atomic energy levels will split symmetrically, $E \rightarrow E \pm t$ forming bonding and antibonding states. The symmetric and antisymmetric modes will be lowered and raised in energy: to a first approximation the shifts are of equal and opposite sign; those occupying the lower energy level are responsible for the chemical bonding. Two helium atoms do not form an electronic bond as both the upper and lower levels are equally populated.

The electromagnetic dipole modes of two helium atoms in close proximity also split, but if we consider the zeropoint energy, all modes are occupied. So why is the Casimir force attractive? It is the second order derivative controlling the time dependence in the equations governing the electromagnetic fields, which constitutes the important difference compared to the first order time derivative of the time-dependent Schrödinger equation, although their spatial parts are similar. As such, the frequency dependence appears as $\omega^{2}$ for harmonic fields after a Fourier transform. When eigenmodes are coupled together due to interaction, it is this quantity which is approximately equally split by the interaction of levels: $\omega^{2} \pm \Delta$ so that even though each mode is equally populated as in the electronic case, there is a net lowering of energy of $-\hbar \Delta^{2} / 8 \omega^{3}$ after taking a square root. This applies to any splitting of degenerate electromagnetic modes such as those between two spheres as discussed above [3], two plasmonic surfaces in proximity [4], or the corrugation of a plasmonic surface where a band gap is opened [5], accounting for the almost universal attractive nature of dispersion forces. Exceptions can occur where the interaction is not between degenerate levels [6].

When a surface is periodically distorted, band gaps are opened and the ensuing asymmetric splitting of levels [3,4] will favor distortion, leading to structural instability. Other more powerful forces may oppose distortion, but we show that Casimir instability sometimes wins. Recent development of nanofabrication and measurement techniques already bring some elaborate designs of fluctuation-related phenomena into reality [6-14], such as Casimir torque [12], nonmonotonic Casimir force in gratings [13], and strong Casimir force reduction [14]. These works reveal that geometric modifications offer an excellent platform to manipulate Casimir forces. However, theoretical studies of Casimir force and energy require a reliable and tractable method to calculate their electromagnetic scattering matrices [15-17]. Owing to its computational speed and accuracy, we employ a method based on transformation optics to investigate the role of Casimir energy and demonstrate that asymmetric splitting of plasmonic modes makes a negative contribution to the surface energy of a single surface. Introducing a second surface boosts the asymmetric splitting and the negative Casimir contribution creates instabilities for both a gold cavity system and a mercury thin film.

Asymmetric splits of plasmonic modes.-We first introduce a conformal mapping technique that can calculate the scattering matrix of corrugated surfaces very efficiently [5] (see Sec. I in Supplemental Material for details [18]). We start from a single corrugated surface with its profile given by a conformal mapping $z=\Gamma \ln \left(e^{w}-i w_{0}\right)^{-1}$, which transforms a flat surface (with the air-metal boundary at $u=u_{0}$ in the slab frame as shown in Fig. 1(a) to a corrugated surface. Here, $w=u_{0}+i v$ and $z=x+i y$ 
(a)
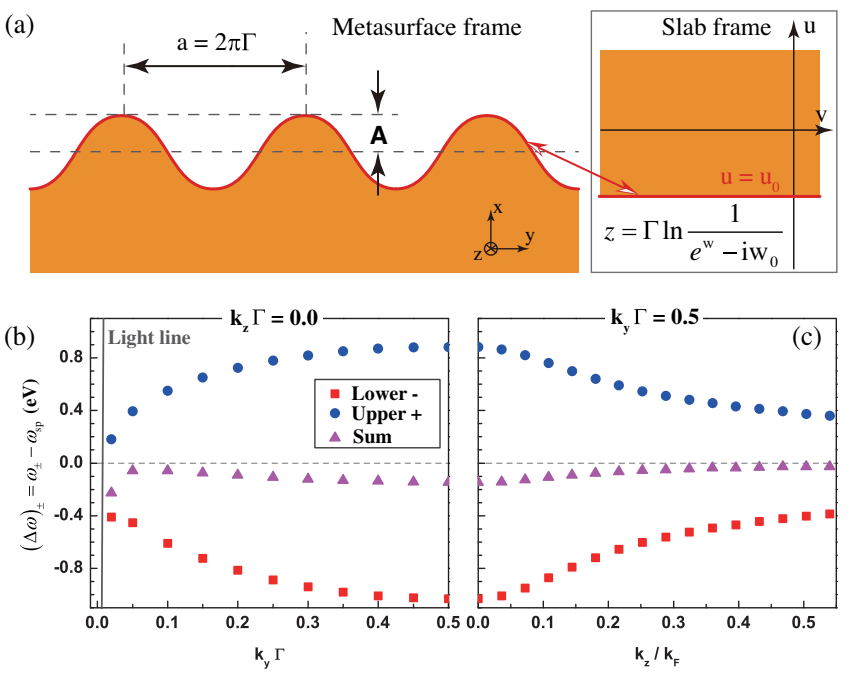

FIG. 1. (a) The schematic picture of a corrugated surface with its period $a$ and modulation strength $A$ defined herein. Its profile in the metasurface frame is generated from the slab frame using a conformal mapping. (b)-(c) The eigenfrequencies of two plasmonic modes for a corrugated surface with respect to $\omega_{\mathrm{sp}}$ in the $k_{z} \Gamma=0.0$ plane (b) and $k_{y} \Gamma=0.5$ plane (c) are plotted by filled squares and circles. The magenta triangles show the sum of these two modes. The geometric parameters are $\Gamma=2.291 \AA$, $w_{0}=0.557$, and $u_{0}=0$. We use a Drude model with $\omega_{p}=9.0 \mathrm{eV}$ and $\gamma=1 \mathrm{e}-4 \mathrm{meV}$.

are the surface coordinates in the slab and metasurface frame, respectively. As shown in Fig. 1(a), the parameter $\Gamma$ determines the corrugation period $a=2 \pi \Gamma$, and the modulation strength $A$, defined as the distance between the top of the protrusion and the average position, is proportional to $w_{0}\left(\left|w_{0}\right| \leq e^{u_{0}}\right)$. When $w_{0}$ is small, the profile of the corrugated surface is almost a sinusoidal function with $A=\Gamma w_{0} / e^{u_{0}}$. We use the Drude model to describe the metal throughout this work.

To confirm the asymmetric split of plasmonic modes, we calculate the eigenfrequencies of the plasmonic modes for the corrugated surface by finding the zeros of $\operatorname{det}\left(R_{1}^{-1}\right)$ in the real frequency axis for a given wave vector. Here, $R_{1}$ is the reflection matrix of the surface in Fig. 1(a) using a Fourier representation. The dimension of $R_{1}$ is $2\left(2 n_{\mathrm{cg}}+1\right)$, where $n_{\mathrm{cg}}$ is the cutoff of the basis of Bloch wavenumber $G=2 \pi / a$, and the factor 2 outside the bracket accounts for two polarizations. Low-index gold surfaces reconstruct to rather complex surface unit cells [32-35], and in order to investigate whether the asymmetric splits of plasmonic modes play a role in the reconstruction, we choose our geometric parameters to mimic the surface profile of the reconstructed $\mathrm{Au}(100)$ surface with a corrugation period $a=14.4 \AA$ and modulation strength $A=1.44 \AA$ [32]. Figures 1(b)-1(c) shows the plasmonic dispersion of such a corrugated plasmonic surface (subtracting the surface plasmon frequency $\omega_{\mathrm{sp}}$ ) and we note that the corrugation splits the plasmonic modes to lower (red squares) and upper (blue circles) plasmonic branches. The sum of their frequencies is shown by magenta dots in Figs. 1(b)-1(c). We see that in the $k_{z} \Gamma=0.0$ plane, the downshift in frequency of the lower branch is larger than the upshift of the upper branch, indicating that their sum is negative with respect to $2 \omega_{\mathrm{sp}}$. This confirms the aforementioned argument that the second order time derivative in Maxwell equations makes the sum of $\omega$ negative. Such asymmetric split increases when $k_{y}$ approaches the Brillouin zone (BZ) boundary [Fig. 1(b)]. Along the homogeneous $z$ direction, $k_{z}$ increases without bound in a continuum model. However, the corrugation-induced split becomes almost symmetric when $k_{z}$ increases to a large value, as shown in Fig. 1(c). Two plasmonic modes and the sum of their frequencies as a function of $k_{z}$ for $k_{y} \Gamma=0.5$ are shown in Fig. 1(c). $k_{z}$ is normalized by the Fermi wave number $k_{F}$, and its value is $k_{F} \Gamma=2.78$. We see that the asymmetric split approaches zero when $k_{z}>0.3 k_{F}$, indicating that the corrugation induced splitting effect rapidly approaches zero when $k_{z}$ is large.

Plasmonic contributions to reconstructed gold surfaces.-The difference in plasmonic zero-point energies should be considered when we consider the change of the ground state surface energy during a surface reconstruction process. We see that the corrugation induced asymmetric splits of plasmonic modes are significant in a certain region of the BZ, and in order to quantify such plasmonic mode contributions to the zero-point energy (per unit area), we use the following expression:

$$
\begin{gathered}
E=\frac{1}{(2 \pi)^{2}} \int_{-\pi / a}^{+\pi / a} d k_{y} \int_{-k_{z c}}^{+k_{z c}} d k_{z} \frac{\hbar}{2 \pi} \int_{0}^{\omega_{p}} d \omega K_{\mathrm{sg}}\left(\omega, k_{y}, k_{z}\right) \\
K_{\mathrm{sg}}\left(\omega, k_{y}, k_{z}\right)=\sum_{i} \operatorname{Im}\left[\ln \left(\lambda^{i}\right)\right]
\end{gathered}
$$

where $\lambda^{i}$ are eigenvalues of reflection matrix $R_{1}$ and $k_{z c}$ is the chosen cutoff value of $k_{z}$. Equation (2) and $\operatorname{det}\left(R_{1}^{-1}\right)$ do essentially the same task of capturing the plasmonic modes in the complex frequency plane $[19,20]$ (see Sec. II A in the Supplemental Material [18]). We plot in Fig. 2(a) the calculated $K_{\mathrm{sg}}$ as a function of $\omega$ for the flat (dashed lines) and corrugated gold (solid lines) surfaces. When the loss is small $\left(\gamma=1 \times 10^{-4} \mathrm{meV}\right)$, the $K_{\mathrm{sg}}$ for the flat surface is a step function, with the sharp change occurring at $\omega_{\mathrm{sp}}$, as shown by the gray dashed line in Fig. 2(a). Corrugations induce two additional steplike features [solid gray line in Fig. 2(a)], indicating the emergence of two plasmonic modes in accordance with the results in Fig. 1. Increasing $\gamma$ to $35 \mathrm{meV}$, the step edges are smoothed, but the salient features of plasmonic modes remain, as shown by the blue lines in Fig. 2(a). This confirms that the $K_{\text {sg }}$ defined in 

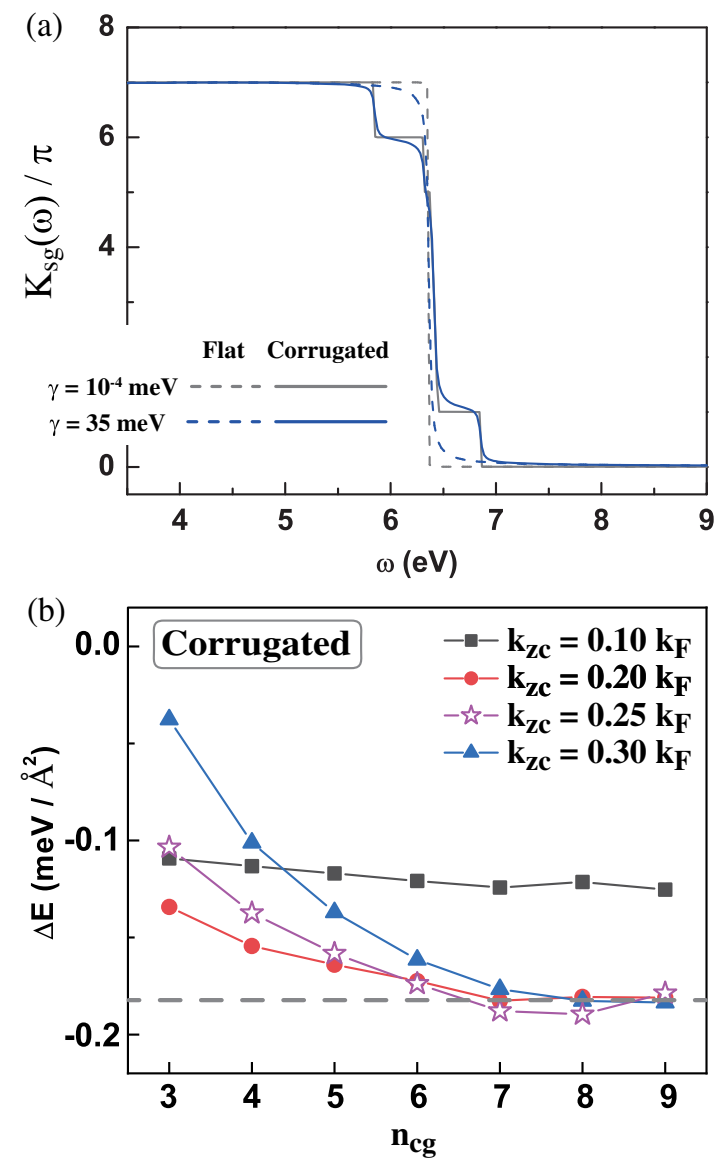

FIG. 2. (a) The $K_{\mathrm{sg}}$ as a function of frequency for $k_{y} \Gamma=0.1$ and $k_{z} \Gamma=-0.8$. The cutoff in Fourier order is $n_{\mathrm{cg}}=3$, which already reaches convergence. The gray and blue lines correspond to $\gamma=1 \times 10^{-4} \mathrm{meV}$ and $\gamma=35 \mathrm{meV}$, respectively. (b) The decreases of surface energy for four different cutoff values of $k_{\mathrm{zc}}$ are shown by solid gray, red, magenta, and blue lines. The dashed line highlights the convergent value $-0.18 \mathrm{meV} / \AA^{2}$.

Eq. (2) successfully captures the plasmonic modes of these surfaces.

We then perform the integral (1) to investigate sum of plasmonic mode frequencies up to the bulk plasmon frequency $\omega_{p}$. As a control calculation, we confirm that the kernel function and integral method successfully reproduces the essential physics for a flat gold surface (See Sec. II B in the Supplemental Material [18]). We then apply Eq. (1) to investigate the zero-point energy plasmonic contributions of a corrugated surface. Figure 2(b) shows the corrugation induced change of zero-point energy $\Delta E[=$ $\left.E\left(w_{0}\right)-E_{0}\right]$ for different values of $k_{\mathrm{zc}}$. For a fixed cutoff $k_{\mathrm{zc}}$, the decrease of zero-point energy converges to a finite number with the increase of $n_{\mathrm{cg}}(>6)$, indicating that the energy differences between flat and corrugated surfaces approach a converged result for a reasonable number of Fourier components along the corrugation $(y)$ direction, and then the only physically meaningful cutoff is $k_{\mathrm{zc}}$. The asymmetric split of plasmonic modes shown in Fig. 1 (a)
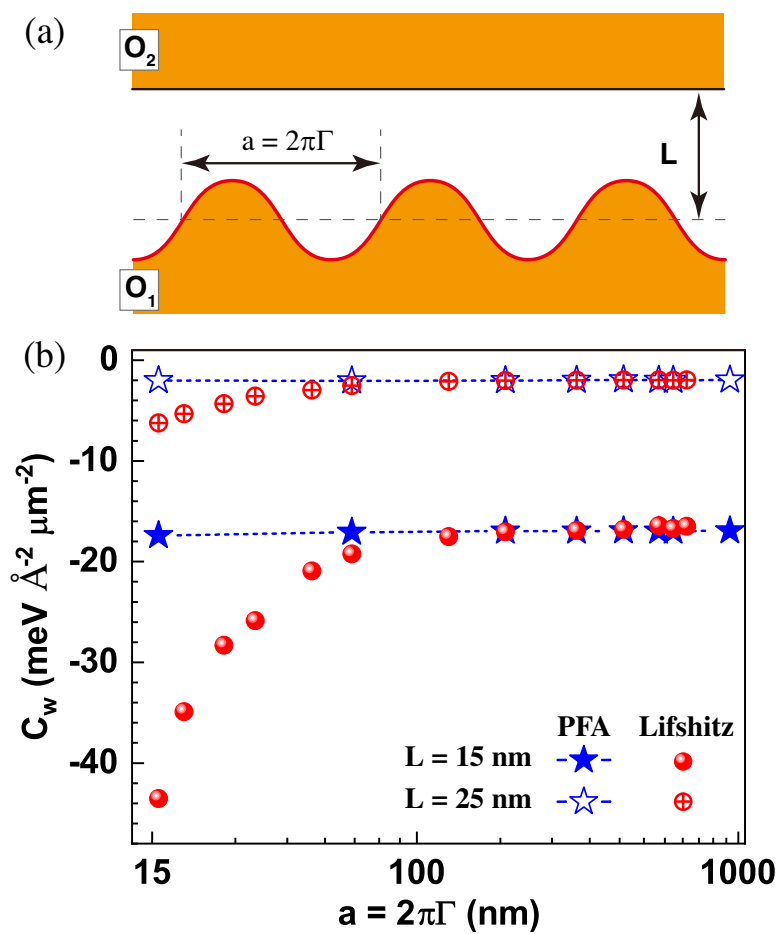

FIG. 3. (a) The schematic picture of the metallic cavity with one corrugated surface and one flat surface. (b) The coefficients $C_{w}$ as a function of the corrugation period $a$ calculated by PFA and Lifshitz formula are shown by blue stars and red circles, respectively. The filled and open markers are results for $L=$ 15 and $25 \mathrm{~nm}$, respectively.

suggests that the decrease of surface energy should approach a finite number when $k_{z}$ is large enough, which is confirmed in Fig. 2(b). We see that the change of zero-point energy converges to $-0.18 \mathrm{meV} / \AA^{2}$ when $k_{\mathrm{zc}}>0.2 k_{F}$, confirming that the splitting of plasmonic modes under geometric corrugations decreases the zero-point energy. We note that there is always a momentum cutoff above which we need to go beyond a continuum model, and we need to solve the plasmon problem using quantum many-body formulations. For $\mathrm{Au}$, that cutoff is $\sim 0.6 k_{F}$ (see Sec. II C in Supplemental Material [18]), and the classical approach here converges (at $k_{\mathrm{zc}} \sim 0.2 k_{F}$ ) well before this limit.

Casimir energy of a metallic cavity.-We see that the zero-point energy of a corrugated surface is lower than that of flat surface due to the asymmetric splitting of plasmonic modes. However, the decrease is small compared with the typical surface energy change in a reconstruction process, e.g., $26.5 \mathrm{meV} / \AA^{2}$ for the $\mathrm{Au}(100)$ surface [32], and hence the zero-point energy of a single air-metal boundary cannot drive metal surface reconstruction. We will show below that Casimir energy change can induce surface corrugation in a metal cavity system defined in Fig. 3(a). The distance $L$ between two surfaces is defined based on the average position. Before performing rigorous calculations, we first 
estimate the role of corrugations under the proximity force approximation (PFA) [16,36]. PFA calculates the Casimir energy by discretizing the corrugated surface into several small flat sectors and add the plane-plane Casimir energy contributions together to give the total energy [21-27]. The fact that the Casimir energy between two perfect mirrors is proportional to $-L^{-3}$ can give us an intuitive understanding of the corrugation effect. Since the dependence of Casimir energy is not linear in $L$, the protruded portion lowers the Casimir energy more than the increase of energy of the concave region, giving an overall decrease of Casimir energy when corrugations are present. To characterize this, we define the quantity $C_{w}$ as $E\left(w_{0}\right)=E_{0}+C_{w} A^{2}$, where $E_{0}$ is the Casimir energy for the cavity containing two flat surfaces $\left(w_{0}=0\right)$ and $E\left(w_{0}\right)$ is that of the cavity with one corrugated surface $\left(w_{0} \neq 0\right)$ with $L$ fixed. Symmetry requires that there is no linear term in $A$ (see Sec. III in the Supplemental Material [18]). The calculated $C_{w}$ by PFA as a function of $a$ for $L=15$ and $25 \mathrm{~nm}$ is shown by filled and open stars in Fig. 3(b). We see that $C_{w}$ is negative for all $a$, and the magnitude of $C_{w}$ for $L=15 \mathrm{~nm}$ is larger than those for $L=25 \mathrm{~nm}$, consistent with the intuitive understanding. We also note that $C_{w}$ does not change much when varying $a$ owing to the way PFA handles the problem.

We then calculate the corrugation induced change in Casimir energy rigorously using the conformal mapping technique. The Casimir energy of such a cavity system can be calculated using the Lifshitz mode counting function $K_{\mathrm{cv}}\left(\omega=i \xi, k_{y}, k_{z}\right)=\ln \operatorname{det}\left[\mathbf{I}-\mathbf{R}_{1} \mathbf{P} \mathbf{R}_{2} \mathbf{P}\right]$ in the integral (1) without any cutoffs in frequency and wave numbers, where $R_{1}$ and $R_{2}$ are reflection matrices of surface $\mathrm{O}_{1}$ and $\mathrm{O}_{2}$ using the Fourier basis and $P_{g g^{\prime}}=\exp \left(i k_{g x} L\right) \delta_{g g^{\prime}}$ is the propagation phase matrix. In our formulation, the integral is performed as usual in the imaginary frequency axis. The calculated $C_{w}$ for $L=15$ and $25 \mathrm{~nm}$ are shown by filled and open red dots in Fig. 3(b). When $a$ is large as compared to $A$, the PFA results agree well with the rigorous Lifshitz result. This shows that PFA can give reasonably reliable Casimir energies when the modulation period is in the micron scale. However, corrugation always increases the surface area, and the surface energy due to bond breaking must increase correspondingly. Whether a metal surface can lower its surface energy by corrugation is then a competition between the decrease due to Casimir energy [Fig. 3(b)] and the increase due to electronic surface energy. We can express the change of energy to the leading order as $\Delta E=\left(C_{w}+C_{\mathrm{sf}}\right) A^{2}$, where $C_{w}$ and $C_{\mathrm{sf}}$ are contributions from Casimir energy and intrinsic surface energy, respectively. The intrinsic surface energy contribution $C_{\mathrm{sf}}$ is proportional to the increase of surface area and equal to $2 \pi^{2} \gamma_{\mathrm{sf}} / a^{2}$, where $\gamma_{\mathrm{sf}}$ is the surface tension coefficient. $C_{\mathrm{sf}}$ is positive and decays parabolically in $a$, while the Casimir energy contribution $C_{w}$ is almost a negative constant for $a$ in the micron scale. Therefore, the surface will be corrugated if another surface $\mathrm{O}_{2}$ is placed close enough to the surface.

It is important to note that the values of $C_{w}$ calculated by the Lifshitz formula are much more negative than those by PFA when the modulation period is less than $100 \mathrm{~nm}$, indicating that corrugations induce a Casimir energy decrease more rapidly than pairwise additive estimations like the PFA. This shows that the surface will be MORE corrugated than the results predicted by PFA because of the additional lowering in energy that is computed accurately by our method. Such a disagreement is partly because PFA ignores the corrugation-induced asymmetric splits in plasmonic modes of a single surface. Figure 3 shows the zeropoint energy lowering due to the coupling with another surface, while the result in Fig. 2 is an intrinsic single boundary lowering in zero-point energy. Both of them share the same mechanism if we understand them as the $\omega^{2}$ eigenvalue perturbation problem.

Instabilities of a mercury film.-We now consider another example of a pair of metal-dielectric boundaries. We consider the stability of a mercury film with a thickness $d$, embedded between two dielectric media $\varepsilon_{1}$ and $\varepsilon_{2}$ (see inset of Fig. 4). We assume that the upper surface has a corrugated profile $A \sin (2 \pi y / a)$, and again express the change of energy as $\Delta E=\left(C_{w}+C_{\mathrm{sf}}\right) A^{2}$, where $C_{w}$ here is the contribution from the Casimir energy change due to the coupling of the plasmons at the two metal-dielectric interfaces. As $C_{\mathrm{sf}}$ is positive and decays parabolically in $a$, while $C_{w}$ is almost a negative constant for the micron size $a$, a mercury film with a given thickness will become unstable because the Casimir energy will dominate when the corrugation period $a$ is larger than a critical value. To quantify the instability, we calculate $C_{w}$ within PFA

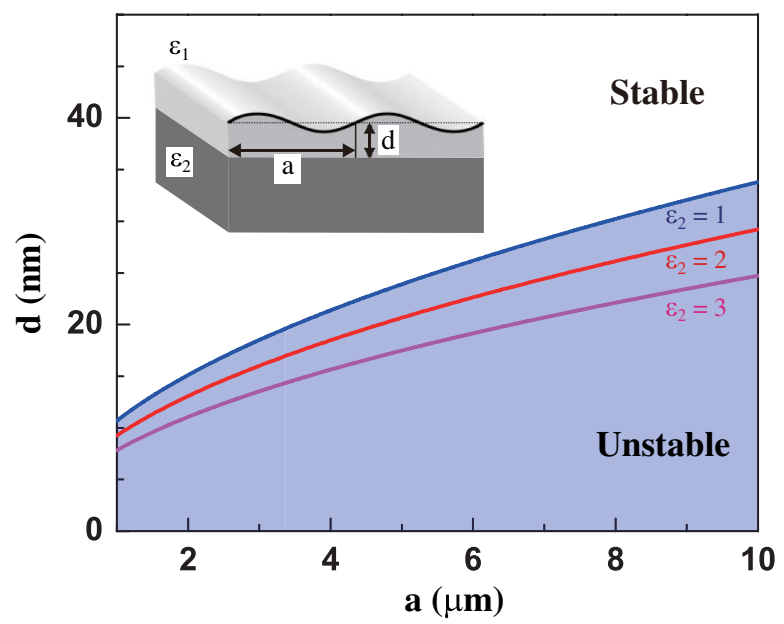

FIG. 4. The $C_{w}+C_{\mathrm{sf}}=0$ line in the thickness $d$ and period $a$ plane for $\varepsilon_{2}=1,2$, and 3 are shown by solid blue, red, and magenta lines, respectively. The inset is the schematic picture of the thin film embedded between two dielectric media $\varepsilon_{1}$ and $\varepsilon_{2}$. The parameters of mercury used are $\omega_{\mathrm{sp}}=6.83 \mathrm{eV}$, and surface tension coefficient $\gamma_{\mathrm{sf}}=27.6 \mathrm{meV} / \AA^{2}$ taken from Ref. [40]. 
together with quasistatic approximation and then plot the $C_{w}+C_{\mathrm{sf}}=0$ line by the solid blue line in Fig. 4 for $\varepsilon_{1}=\varepsilon_{2}=1$, which confirms the above statements [37]. The mercury film is usually placed on some substrate, so we also plot the $C_{w}+C_{\text {sf }}=0$ line for $\varepsilon_{2}=2$ and 3 by the solid red and magenta line, respectively, while keeping $\varepsilon_{1}=1$. Compared with the $\varepsilon_{2}=1$ case, we see that the critical values of $a$ increase as $\varepsilon_{2}$ increases. All these results show that a flat thin mercury film is unstable against corrugation in the large scale. In other words, the Casimir energy contributed by surface plasmons due to surface corrugations provides a fundamental scale limit to planar nanostructures. This is also an example of a RayleighTaylor instability $[38,39]$.

Conclusions.-We demonstrated that surface corrugations induce plasmonic modes of metal surfaces to split unevenly because the equation of motion of plasmons is second order in time which is generally true for electromagnetic excitations. Such an asymmetric split always decreases the zero-point energy of a single air-metal interface and can give rises to the decrease of the Casimir energy for a cavity system consisting of two metallic surfaces. It also contributes to the instability of a mercury thin film against corrugation. Since such an asymmetric mode splitting is intrinsic to the coupling of plasmonic modes, their contributions can be observed in other hybridized plasmonic systems, leading to one kind of electromagnetic bonding, and can cause the attraction of neutral metallic objects. Last but not least, accurate calculations of zero-point and Casimir energies of complex interfaces usually require a formidable amount of computer resources, and our calculations are made tractable by the conformal mapping technique which can provide an excellent platform to investigate other electromagnetic fluctuation-type problems.

K. D. and J. B. P. acknowledge support from the Gordon and Betty Moore Foundation. D. O. acknowledges support from the Imperial College President's Scholarship. K. D. and C. T. C. acknowledge funding from Research Grants Council (RGC) Hong Kong through Grants No. AoE/P-02/ 12 and No. 16303119.

*phchan@ust.hk

j.pendry@imperial.ac.uk

[1] J. N. Israelachvili, Intermolecular and Surface Forces (Academic, San Diego, 1998).

[2] A. W. Rodriguez, F. Capasso, and S. G. Johnson, Nat. Photonics 5, 211 (2011).

[3] P. Nordlander, C. Oubre, E. Prodan, K. Li, and M. I. Stockman, Nano Lett. 4, 899 (2004).

[4] F. Intravaia, C. Henkel, and A. Lambrecht, Phys. Rev. A 76, 033820 (2007).

[5] J. B. Pendry, P. A. Huidobro, and K. Ding, Phys. Rev. B 99, 085408 (2019).
[6] R. Zhao, L. Li, S. Yang, W. Bao, Y. Xia, P. Ashby, Y. Wang, and X. Zhang, Science 364, 984 (2019).

[7] S. K. Lamoreaux, Phys. Rev. Lett. 78, 5 (1997).

[8] H. B. Chan, Y. Bao, J. Zou, R. A. Cirelli, F. Klemens, W. M. Mansfield, and C. S. Pai, Phys. Rev. Lett. 101, 030401 (2008).

[9] A. Lambrecht and V. N. Marachevsky, Phys. Rev. Lett. 101, 160403 (2008).

[10] J. N. Munday, F. Capasso, and V. A. Parsegian, Nature (London) 457, 170 (2009).

[11] A. A. Banishev, J. Wagner, T. Emig, R. Zandi, and U. Mohideen, Phys. Rev. Lett. 110, 250403 (2013).

[12] D. A. T. Somers, J. L. Garrett, K. J. Palm, and J. N. Munday, Nature (London) 564, 386 (2018).

[13] L. Tang, M. Wang, C. Y. Ng, M. Nikolic, C. T. Chan, A. W. Rodriguez, and H. B. Chan, Nat. Photonics 11, 97 (2017).

[14] F. Intravaia, S. Koev, I. W. Jung, A. A. Talin, P. S. Davids, R. S. Decca, V. A. Aksyuk, D. A. R. Dalvit, and D. Lopez, Nat. Commun. 4, 2515 (2013).

[15] L. M. Woods, D. A. R. Dalvit, A. Tkatchenko, P. RodriguezLopez, A. W. Rodriguez, and R. Podgornik, Rev. Mod. Phys. 88, 045003 (2016).

[16] A. Lambrecht, P. A. Maia Neto, and S. Reynaud, New J. Phys. 8, 243 (2006).

[17] M. T. H. Reid, A. W. Rodriguez, J. White, and S. G. Johnson, Phys. Rev. Lett. 103, 040401 (2009).

[18] See Supplemental Materials at http://link.aps.org/ supplemental/10.1103/PhysRevLett.126.046802 for numerical details, which includes Refs. [5,19-31].

[19] J. B. Pendry and L. M. Moreno, Phys. Rev. B 50, 5062 (1994).

[20] F. Wijnands, J. B. Pendry, F. J. Garcia-Vidal, P. M. Bell, P. J. Roberts, and L. Martin Moreno, Opt. Quantum Electron. 29, 199 (1997).

[21] D. E. Krause, R. S. Decca, D. López, and E. Fischbach, Phys. Rev. Lett. 98, 050403 (2007).

[22] H. Gies and K. Klingmüller, Phys. Rev. Lett. 96, 220401 (2006).

[23] C. D. Fosco, F. C. Lombardo, and F. D. Mazzitelli, Phys. Rev. D 84, 105031 (2011).

[24] L. P. Teo, M. Bordag, and V. Nikolaev, Phys. Rev. D 84, 125037 (2011).

[25] M. Hartmann, G.-L. Ingold, and P. A. Maia Neto, Phys. Rev. Lett. 119, 043901 (2017).

[26] R. B. Rodrigues, P. A. Maia Neto, A. Lambrecht, and S. Reynaud, Phys. Rev. Lett. 96, 100402 (2006).

[27] R. B. Rodrigues, P. A. Maia Neto, A. Lambrecht, and S. Reynaud, Phys. Rev. A 75, 062108 (2007).

[28] M. G. Moharam and T. K. Gaylord, J. Opt. Soc. Am. 73, 451 (1983).

[29] M. G. Moharam, E. B. Grann, D. A. Pommet, and T. K. Gaylord, J. Opt. Soc. Am. A 12, 1068 (1995).

[30] L. Li and C. W. Haggans, J. Opt. Soc. Am. A 10, 1184 (1993).

[31] P. Lalanne and G. M. Morris, J. Opt. Soc. Am. A 13, 779 (1996).

[32] X.-Q. Wang, Phys. Rev. Lett. 67, 3547 (1991).

[33] J. V. Barth, H. Brune, G. Ertl, and R. J. Behm, Phys. Rev. B 42, 9307 (1990).

[34] Ch. Wöll, S. Chiang, R. J. Wilson, and P. H. Lippel, Phys. Rev. B 39, 7988 (1989). 
[35] U. Harten, A. M. Lahee, J. P. Toennies, and Ch. Wöll, Phys. Rev. Lett. 54, 2619 (1985).

[36] B. V. Derjaguin, Kolloid Z. 69, 155 (1934).

[37] N. G. van Kampen, B. R. A. Nijboer, and K. Schram, Phys. Lett. 26A, 307 (1968).
[38] J. W. S. Rayleigh, Proc. London Math. Soc. s1-14, 170 (1883).

[39] G. I. Taylor, Proc. R. Soc. Ser. A 201, 192 (1950).

[40] B.-O. Kim, G. Lee, E. W. Plummer, P. A. Dowben, and A. Liebsch, Phys. Rev. B 52, 6057 (1995). 